\title{
The 2039 A/G FSH receptor gene polymorphism influences glucose metabolism in healthy men
}

\author{
Rossella Cannarella $\mathbb{1}^{1} \cdot$ Nicolò Musso $^{2} \cdot$ Rosita A. Condorelli ${ }^{1} \cdot$ Marco Musmeci $^{1} \cdot$ Stefania Stefani $^{2}$. \\ Antonio Aversa $^{3} \cdot$ Sandro La Vignera ${ }^{1} \cdot$ Aldo E. Calogero $^{1}$
}

Received: 13 April 2020 / Accepted: 6 July 2020 / Published online: 17 July 2020

(C) The Author(s) 2020

\begin{abstract}
Objective To assess the role of c. 2039 A/G (p. Asp680Ser) (rs6166) and c. -29 G/A (rs1394205) follicle-stimulating hormone receptor (FSHR) gene single nucleotide polymorphisms (SNPs) in a cohort of healthy men.

Methods One-hundred twenty-seven healthy men underwent evaluation of the anthropometric parameters, assessment of metabolic and lipid profile, measurement FSH serum levels, and genotyping of both the aforementioned FSHR SNPs. Data grouped according to the FSHR rs6166 or rs1394205 genotypes underwent to statistical analysis.

Main results The three groups of men for each FSHR SNP did not differ statistically significantly for body mass index and serum FSH levels. As for FSHR rs6166 SNP, glucose levels were significantly lower in men with the GG genotype compared with those with the AA genotype. Men with AG had lower insulin levels and HOMA index values compared with those carrying the genotype AA $(p<0.05)$. The GG group showed a negative correlation between serum FSH levels and insulin and between serum FSH levels and HOMA index $(p<0.05)$. In contrast, men grouped according to the FSHR rs1394205 genotype showed no significant difference in blood glucose, serum insulin levels, and HOMA index. The AG group showed a negative correlation between FSH insulin and between serum FSH levels and HOMA index $(p<0.05)$.

Conclusions Men with the genotype GG of the FSHR rs6166 SNP have lower blood glucose levels than those with the AA genotype. Their FSH levels inversely correlated with insulin and HOMA index. In contrast, the genotype FSHR rs6166 A/G did not reveal any role of FSH on glucose metabolism in healthy men. The inverse relationship between FSH and insulin or HOMA index in the group with the genotype GG of the FSHR rs6166 SNP suggests a possible cross-talk between FSH and insulin.
\end{abstract}

Keywords FSH $\cdot 2039$ A/G FSHR $\cdot$ FSHR polymorphism $•$ Glucose $・$ Metabolism $•$ Insulin

\section{Introduction}

The follicle-stimulating hormone (FSH) is a glycoprotein made of an $\alpha$ and a $\beta$ subunit. It exerts its peripheral effects by binding to its receptor (FSHR), a 678 amino acid protein belonging to the G-coupled receptor family, expressed in

Aldo E. Calogero

acaloger@unict.it

1 Department of Clinical and Experimental Medicine, University of Catania, Catania, Italy

2 Bio-nanotech Research and Innovation Tower (BRIT), University of Catania, Catania, Italy

3 Department of Experimental and Clinical Medicine, "Magna Graecia" University, Catanzaro, Italy granulosa and Sertoli cells, where FSH stimulates follicle genesis and spermatogenesis, respectively [1].

The follicle-stimulating hormone receptor (FSHR) gene, mapping in the $2 \mathrm{p} 16.3$ chromosome, contains ten exons and nine introns. The first nine exons encode for the majority of the extracellular domain. The exon 10 encodes for the Cterminal region of the extracellular domain, the transmembrane and the intracellular domains [1]. The HapMap database (http://hapmap.ncbi.nlm.nih.gov) lists more than 900 FSHR single nucleotide polymorphisms (SNPs). A large body of evidence has been released on the c. $2039 \mathrm{~A} /$ G (p. Asp680Ser) (rs6166) and the c. -29 G/A (rs1394205) FSHR SNPs so far. The first maps in the exon 10, which encodes for the transmembrane domain of the FSHR, and it is known to influence the efficiency of signal transduction. In detail, the c. $2039 \mathrm{~A} / \mathrm{G}$ genotype impacts on the expression of the amino acid 680 and the FSHR Ser680Ser 
(GG) results to be more resistant to the FSH signal compared with the Asp680Asp (AA) [2]. The c. -29 G/A SNP maps in the FSHR gene promoter and influences FSHR expression. Particularly, the A allele is associated with a lower (by 56\%) transcription activity of the promoter [3].

Classically, the effects of FSH have been restricted to gonads and a large body of evidence of FSHR SNPs has been published on fertility in both genders [4-6]. However, recent data point to the existence of extra-gonadal effects of FSH. Accordingly, the FSHR is expressed in human bone cells and in human bone marrow adipocytes [7, 8]. Furthermore, FSH has been already addressed as possibly implicated in the postmenopausal changes of bone mineral density [7, 9] and in lipid distribution, adiposity, and metabolism in male patients with hypergonadotropic hypogonadism [8].

To further explore the role that FSH may play in glucose and lipid metabolism in the male gender, we investigated the possible influence of the c. 2039 A/G (p. Asp680Ser) (rs6166) and the c. -29 G/A (rs1394205) FSHR SNPs on blood glucose, insulin, total, HDL and LDL cholesterol, triglycerides, on the homeostasis model assessment (HOMA) index and the body mass index (BMI) in a cohort of healthy men.

\section{Subjects and methods}

\section{Patient selection}

The study has been carried out in male patients referring to the Division of Andrology and Endocrinology, University of Catania, for an endocrinologic or andrologic counseling. A detailed medical history was collected and weight and height were assessed for each man. The BMI was calculated using the formula: weight $(\mathrm{kg}) /[$ height $(\mathrm{m}) \times$ height $(\mathrm{m})]$. A total of 146 men were evaluated for inclusion in this study. The following exclusion criteria were used and this resulted in the exclusion of a number of men that is indicated in parentheses: azoospermia $(n=3)$, tumor $(n=2)$, assumption of metformin, insulin, hypoglycemic or hypocholesterolemic drugs $(n=5)$, diabetes $(n=2)$, use of gonadotoxic drugs $(n=2)$, hypogonadism $(n=3)$, testosterone replacement therapy $(n=1)$, FSH therapy $(n=1)$, major adverse cardiovascular events $(n=0)$, and major comorbilities $(n=0)$. Taking all this into account, 127 Caucasian men from Eastern Sicily were included in the study.

Hormone evaluation was performed by electro chemiluminescence (Hitachi-Roche equipment, Cobas 6000, Roche Diagnostics, Indianapolis, IN, USA). Reference values were as follows: FSH 0.95-11.95 IU/l, glycemia 74-100 mg/dl, insulin 1.9-23 $\mu \mathrm{IU} / \mathrm{ml}$, total cholesterol $0-200 \mathrm{mg} / \mathrm{dl}$, HDL cholesterol 35-55 mg/dl, LDL cholesterol $<160 \mathrm{mg} / \mathrm{dl}$, and triglycerides $35-200 \mathrm{mg} / \mathrm{dl}$. The HOMA index was evaluated using the formula: [glycemia $(\mathrm{mg} / \mathrm{dl}) \times$ insulin $(\mu \mathrm{IU} /$ $\mathrm{ml})] / 405$. The upper normal value of HOMA index was 2.5 .

\section{FSHR analysis}

Genomic DNA was extracted from blood cells using the PureLink $^{\circledR}$ Genomic DNA Kits (invitrogen Catalog Numbers K1821-04) for purification of genomic DNA according to the manufacturer's instructions. The concentration and the quality of the DNA was determined using a ND-1000 spectrophotometer (NanoDrop, Thermo Scientific, USA). Allelic Discrimination was performed with TaqMan assay in order to show a different genotyping distribution of FSHR polymorphism. Probes and primers for rs6166 FSHR and rs1394205 FSHR polymorphisms were chosen on https://www.thermofisher.com/it/en/home/life-science/pcr/ real-time-pcr/real-time-pcr-assays/snp-genotyping-taqman-a ssays.html?SID=fr-taqman-2. The reaction was carried out according to manufacturer's instructions (cod 4371355, Applied Biosystems, CA, USA). Each DNA sample was analyzed in triplicate [10]. Allelic Discrimination real-time PCR analysis was performed using LightCycler ${ }^{\circledR} 480$ System (Roche Molecular Systems, Inc).

\section{Statistical analysis}

The normality of the variables was evaluated with the Shapiro-Wilk test. Descriptive statistical results have been reported as mean \pm standard deviation (SD) for not-skewed variables. Statistical analysis was performed by one-way analysis of variance, followed by the Duncan's Multiple Range Test, using SPSS 22.0 for Windows (SPSS Inc., Chicago, IL, USA). For correlation analysis, the Pearson or the Kendall tests were used, according to the data distribution. A $p$ value $<0.05$ was accepted as statistically significant.

\section{Results}

The number and the prevalence (in parentheses) of FSHR rs6166 genotypes were: 33 (26.0\%), 63 (49.6\%), and 31 (24.4\%) for AA, AG, and GG, respectively. For the genotype FSHR rs1394205, they were: 14 (11.0\%), 38 (29.9\%), and $75(59.1 \%)$ for AA, AG, and GG genotypes, respectively. The FSHR allelic frequencies and the estimated $F S H R$ haplotype frequencies found in this cohort were consistent with previously published data from unrelated Caucasian populations [9, 11-13].

Data were normally distributed. Anthropometric data and biochemical parameters of men grouped according to the 
Table 1 Antropometric data and biochemical parameters in 127 healthy men classified according to rs6166 or rs 1394205 polymorphisms of the FSH receptor gene

\begin{tabular}{|c|c|c|c|c|c|c|}
\hline \multirow[t]{2}{*}{ Parameters } & \multicolumn{3}{|l|}{ rs6166 } & \multicolumn{3}{|l|}{ rs1394205 } \\
\hline & AA & AG & GG & AA & AG & GG \\
\hline Number & 33 & 63 & 31 & 14 & 38 & 75 \\
\hline Age (years) & $37.0 \pm 8.7$ & $36.6 \pm 9.2$ & $35.7 \pm 7.6$ & $40.4 \pm 8.5$ & $36.9 \pm 10.9$ & $35.4 \pm 7.4$ \\
\hline BMI $\left(\mathrm{kg} / \mathrm{m}^{2}\right)$ & $27.0 \pm 4.9$ & $27.3 \pm 5.4$ & $26.5 \pm 3.8$ & $27.0 \pm 3.4$ & $26.6 \pm 5.5$ & $27.1 \pm 4.9$ \\
\hline Glycaemia (mg/dl) & $90.5 \pm 7.4$ & $86.2 \pm 8.6$ & $82.2 \pm 5.5^{*}$ & $87.4 \pm 12.7$ & $88.2 \pm 7.9$ & $85.2 \pm 7.8$ \\
\hline Insulin $(\mu \mathrm{IU} / \mathrm{ml})$ & $11.0 \pm 4.8$ & $7.3 \pm 3.0 *$ & $9.0 \pm 1.4$ & $7.5 \pm 2.9$ & $8.1 \pm 3.7$ & $9.4 \pm 3.5$ \\
\hline HOMA index & $2.5 \pm 1.3$ & $1.6 \pm 0.7 *$ & $1.8 \pm 1.0$ & $1.6 \pm 0.9$ & $1.8 \pm 0.9$ & $2.0 \pm 1.0$ \\
\hline Cholesterol (mg/dl) & $184.7 \pm 46.6$ & $179.4 \pm 36.6$ & $178.6 \pm 30.1$ & $208.5 \pm 45.5$ & $174.3 \pm 28.6^{*}$ & $184.1 \pm 37.7$ \\
\hline HDL cholesterol (mg/dl) & $46.8 \pm 14.2$ & $50.4 \pm 13.6$ & $47.0 \pm 9.3$ & $52.2 \pm 12.8$ & $49.7 \pm 10.0$ & $46.9 \pm 11.7$ \\
\hline LDL cholesterol (mg/dl) & $134.4 \pm 46.6$ & $115.8 \pm 32.1$ & $120.9 \pm 31.1$ & $145.3 \pm 40.7$ & $115.7 \pm 39.8$ & $121.6 \pm 32.7$ \\
\hline Triglycerides (mg/dl) & $88.6 \pm 49.3$ & $81.4 \pm 64.4$ & $93.1 \pm 40.2$ & $165.8 \pm 33.7$ & $92.5 \pm 57.2$ & $92.2 \pm 63.1$ \\
\hline FSH (IU/l) & $5.0 \pm 3.2$ & $4.5 \pm 2.9$ & $3.9 \pm 2.5$ & $4.0 \pm 2.3$ & $4.5 \pm 3.0$ & $4.4 \pm 2.8$ \\
\hline
\end{tabular}

Data are expressed as mean \pm standard deviation for continual variables. The $p$ values were calculated using ANOVA for continuous variables

FSH follicle-stimulating hormone, BMI body mass index, HOMA homeostasis model assessment ${ }^{*} p<0.05$ vs. AA (ANOVA followed by Duncan Multiple Range Test)
FSHR rs6166 or rs1394205 genotype are shown in Table 1. No significant difference in all parameters was observed between men classified according to the different genotype ( $p>0.05$ in all cases), with the exception of total cholesterol, which was higher in men with FSHR rs1394205 AA genotype compared with those with the AG genotype $(p<$ 0.05). According to their BMI, $42.2 \%$ of patients were overweight, $20 \%$ obese; $8.3 \%$ had hyperglicemia, $20.5 \%$ insulin resistance, and $26.3 \%$ hypercholesterolemia. The prevalence of these features did not differ after classification by the FSHR genotype.

Serum glucose levels were lower in men with FSHR rs6166 GG vs. men with the AA genotype. Men with AG SNP had lower insulin levels and HOMA index values (Table 1) compared with AA $(p<0.05)$. Men grouped for the FSHR rs1394205 genotype did not show any difference in glycaemia, insulin, and HOMA index (Fig. 1).

No significant correlation was found between FSH and glycaemia $(r=0.01)$, insulin $(r=-0.26)$ or HOMA index $(r=-0.19)$ in the entire cohort. Correlation analysis in men grouped by FSHR rs6166 or rs1394205 genotypes are shown in Table 2. A significant negative correlation between FSH and insulin and FSH and HOMA index was observed in men with the genotype FSHR rs6166 GG and for the genotype FSHR rs1394205 AG. All the other correlations were not significant.

\section{Discussion}

The results of the present study showed that the polymorphism rs6166 in exon 10 of the FSHR gene influences significantly blood glucose levels in healthy men. Particularly, the GG genotype, encoding for a less efficiently transducing FSHR protein, is associated with lower glucose levels compared to the AA one, a SNP that more efficiently transduce the FSH signal. Since the groups resulted to have a similar age, BMI and FSH levels, these findings suggest that the FSHR gene plays a role on glucose metabolism in men. The significant lower insulin levels and HOMA index in men with the AG genotype compared those with AA suggest a sort of compensatory mechanism to avoid hyperglycemia, which is not observed in men with GG due to the normal glucose levels. Interestingly, an inverse relationship between FSH and insulin levels or HOMA index was found in men with the genotype GG. Furthermore, no effect on such parameters was observed when men were classified according to the rs1394205 genotype, aside a significant indirect correlation between FSH and insulin and a negative trend between FSH and HOMA index in the AG group.

Scanty evidence has been published on the impact of FSH on glucose metabolism so far. A preclinical study reported that female mice FSHR gene deficient $\left(F s h r^{+/-}\right.$ mice) do not develop obesity as wild-type ovariectomized mice do. These effects were also reported in male $F s h r^{+/-}$ mice. Furthermore, the administration of a blocking antibody against FSH was found to efficiently induce thermogenic adipose tissue and to reduce body fat in both sexes [14]. This indicates that FSH may be involved in the development of obesity. In addition, the FSHR has been found expressed in pancreatic $B$ cells in animals but its function in this district is still unclear [15].

A recent clinical study investigated whether FSH may impact on glucose and lipid metabolism in a large number of patients (more then 300) with hypergonadotropic hypogonadism, including patients with Klinefelter syndrome. Remarkably, the authors reported that FSH could enhance soluble-RANKL (sRANKL) secretion from human adipocytes. sRANKL levels, in turn, are negatively associated 

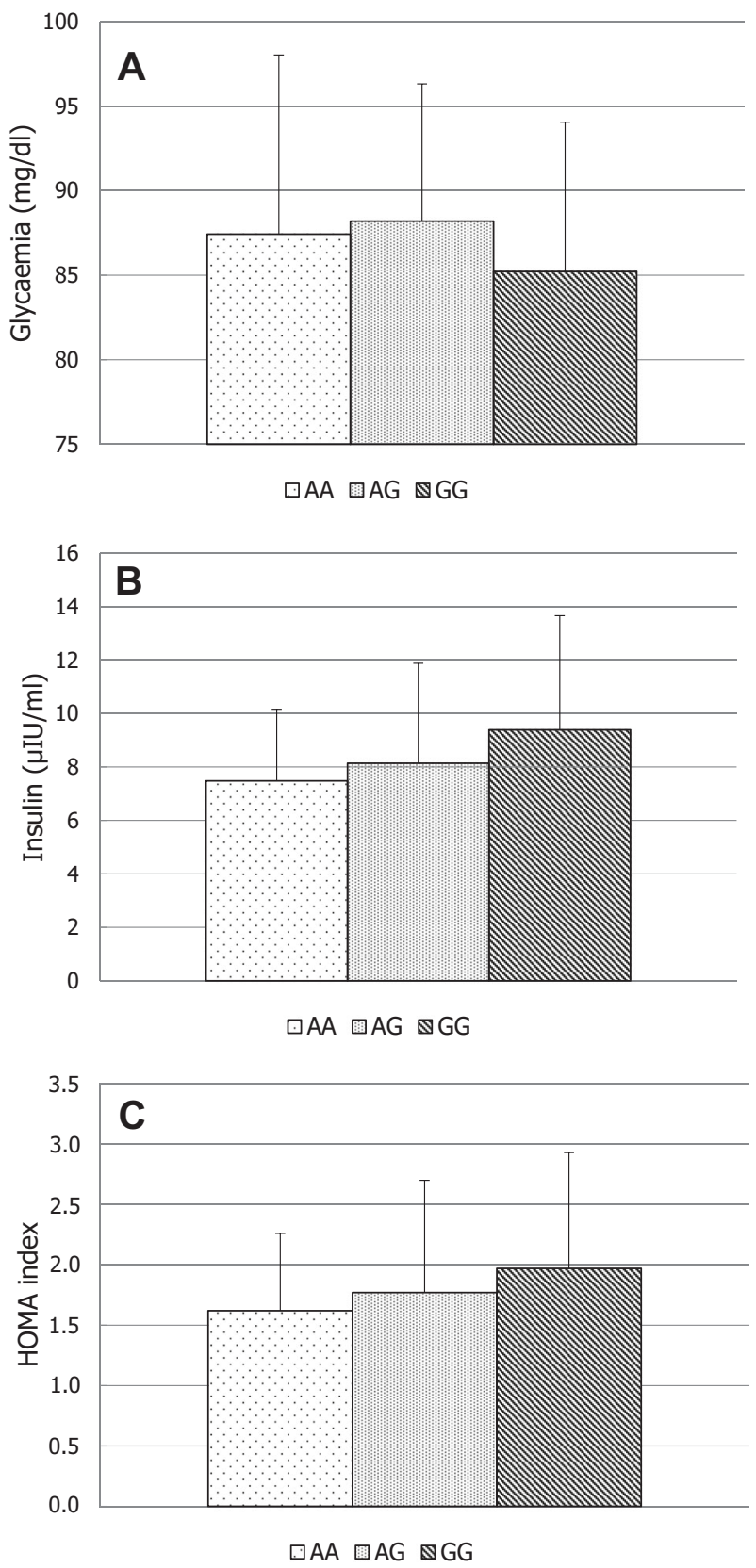

Fig. 1 Glycaemia, insulin, and HOMA index classified according to the rs1394205 FSHR genotype. No significant difference was found in AA, AG, and GG groups for glycaemia (a), insulin (b), and HOMA index $(\mathbf{c})$ with fat percentage, fasting insulin, and glucose [8]. sRANKL has been shown to decrease pancreatic $\beta$ cell proliferation and to decrease glucose tolerance [16]. Accordingly, the administration of the sRANKL inhibitor denosumb improves glucose tolerance, but not HbA1c levels in nondiabetic women $[17,18]$. Thus, the association between FSH and glucose metabolism may be indirect being mediated by the FSH-induced sRANKL production.

The inverse relationship between $\mathrm{FSH}$ and insulin or HOMA index observed in men with the GG genotype of the FSHR rs6166 SNP suggests the hypothesis that FSH and insulin may share a common molecular signaling. Generally, tyrosine kinase receptors (e.g., insulin receptor or insulin-like growth factor receptor) are able to influence the FSHR signaling [19-21]. In this regard, the insulin receptor substrate 1, which represents a co-factors linked to tyrosine kinase receptors, seems to play a role in the pathogenesis of insulin resistance [22, 23]. Interestingly, it has been described as the hub that links FSH signaling pathway and the tyrosine kinase receptor-dependent cascade [24, 25]; thus relating FSHR to the insulin receptor. The possible cross-talk between FSH and insulin has been further investigated in an in vitro study on porcine prepubertal Sertoli cells aimed to evaluate the secretion of anti-Müllerian hormone $(\mathrm{AMH})$ and inhibin in response to $\mathrm{FSH}$ and insulin. The results showed that insulin significantly decreased FSH-stimulated $\mathrm{AMH}$ and inhibin secretion, suggesting that insulin may likely interfere with the FSH signaling [26]. In line with this evidence, a recent clinical study reported that insulin is able to decrease the response of granulosa cells to FSH stimulation in women with obesity-related infertility during in vitro fertilization [27]. Unfortunately, clinical evidence is very limited in men, due to the small duration of FSH stimulation of the poorly standardized protocols used for the treatment of infertile male patients [28]. FSH administration to patients with oligozoospermia, normal serum FSH levels, and insulin resistance showed to increase its efficacy on sperm parameters and fertility outcome when it was co-administered with metformin [29]. These findings add further evidence on the possible interference of insulin in FSH signaling also in men. Conversely, FSH may somehow influence insulin pathway and glucose metabolism, which may be dimly
Table 2 Correlation analysis of glycaemia, insulin, and HOMA index with the rs6166 or rs1394205 polymorphisms of the FSH receptor gene, in 127 healthy men

\begin{tabular}{lllllllll}
\hline Parameters & rs6166 & & & & rs1394205 & \\
\cline { 2 - 3 } & AA & AG & GG & & AA & AG & GG \\
\hline FSH vs. glycaemia & $r=-0.29$ & $r=0.07$ & $r=0.12$ & & $r=0.11$ & $r=-0.20$ & $r=0.08$ \\
FSH vs. insulin & $r=-0.02$ & $r=-0.16$ & $r=-0.69^{*}$ & $r=-0.31$ & $r=-0.67^{*}$ & $r=-0.19$ \\
FSH vs. HOMA index & $r=-0.11$ & $r=-0.07$ & $r=-0.71^{*}$ & $r=-0.17$ & $r=-0.70^{*}$ & $r=-0.07$ \\
\hline
\end{tabular}

HOMA homeostasis model assessment, FSH follicle-stimulating hormone $* p \leq 0.05$ 
supported by the significantly increase of FSH serum levels in diabetic patients, as results of meta-analysis have shown [30]. In addition, an inverse correlation between FSH and insulin, and FSH and HOMA index were found in the rs1394205 AG group. The reason of this result is hardly to be interpreted. This strengthen the importance of fully addressing the molecular relationship between FSHR and insulin.

Finally, the results of the present study expand the view of the possible clinical applications of FSHR SNPs assessment. A possible FSHR SNPs usefulness has been classically searched on fertility so far. However, the research promoted after the identification of FSH effects on bone tissue has led to studies aimed at exploring its protective role against the development of osteoporosis in women with the FSHR rs6166 GG genotype; thus supporting extragonadal effects that FSH may likely have [9]. Similarly, the importance of FSHR SNPs in the metabolic field needs to be further addressed.

Our results need to be taken with care due to the relatively small sample size examined. Hence, further studies on greater cohorts are warranted.

In conclusion, we found significantly lower blood glucose levels in men with the GG genotype of the FSHR rs6166 SNP, and significantly lower insulin levels and HOMA index in AG compared with AA men. Furthermore, FSH serum levels correlated inversely with insulin levels and the HOMA index in men with GG, no effects were observed on these parameters when men were classified according to the rs1394205 genotype, although a significant indirect correlation between FSH and insulin and a negative trend between FSH and HOMA index was found in the AG group. FSHR rs6166 A/G genotypization could reveal the impact of FSH on glucose metabolism in healthy men. The inverse relationship of FSH with insulin and the HOMA index in the FSHR rs6166 GG group may support the existence of a cross-talk between FSH and insulin. Accordingly, the higher is insulin, the lower is FSH, thus reflecting the importance of treating insulin resistance prior to FSH administration in oligozoospermic patients [29]. Molecular mechanisms underlying the interaction between FSH and insulin are still matter of debate. Unpublished data support that incubation with insulin down-regulates FSH receptor in an in vitro culture of porcine Sertoli cells. The in vivo findings of a recently published study [29] may somehow reflect this molecular mechanism. With this in mind, the inverse relationship between FSH and insulin and between FSH and HOMA index in the GG group (encoding for a more resistant FSHR to signal transduction) could confirm the occurrence of a molecular interaction between insulin and the FSHR. Clearly, this topic represents a black hole and deserves further investigation.
Acknowledgements Open access funding provided by Università degli Studi di Catania within the CRUI-CARE Agreement.

\section{Compliance with ethical standards}

Conflict of interest The authors declare that they have no conflict of interest.

Ethical approval All procedures performed in studies involving human participants were in accordance with the ethical standards of the institutional and/or national research committee and with the 1964 Helsinki declaration and its later amendments or comparable ethical standards. This study was approved by the Teaching Hospital "G. Rodolico" Ethics Committee (Protocol n. 178/2017/PO) and informed written consent was obtained from each participant after full explanation of the purpose and nature of all procedures used. The study has been carried out in accordance with the principles expressed in the Declaration of Helsinki.

Informed consent Informed consent was obtained from all individual participants included in the study.

Publisher's note Springer Nature remains neutral with regard to jurisdictional claims in published maps and institutional affiliations.

Open Access This article is licensed under a Creative Commons Attribution 4.0 International License, which permits use, sharing, adaptation, distribution and reproduction in any medium or format, as long as you give appropriate credit to the original author(s) and the source, provide a link to the Creative Commons license, and indicate if changes were made. The images or other third party material in this article are included in the article's Creative Commons license, unless indicated otherwise in a credit line to the material. If material is not included in the article's Creative Commons license and your intended use is not permitted by statutory regulation or exceeds the permitted use, you will need to obtain permission directly from the copyright holder. To view a copy of this license, visit http://creativecommons. org/licenses/by/4.0/.

\section{References}

1. J. Gromoll, E. Pekel, E. Nieschlag, The structure and organization of the human follicle-stimulating hormone receptor (FSHR) gene. Genomics 35, 308-311 (1996)

2. L. Casarini, V. Moriondo, M. Marino, F. Adversi, F. Capodanno, C. Grisolia, A. La Marca, G.B. La Sala, M. Simoni, FSHR polymorphism p.N680S mediates different responses to FSH in vitro. Mol. Cell. Endocrinol. 393, 83-91 (2014)

3. T. Nakayama, N. Kuroi, M. Sano, Y. Tabara, T. Katsuya, T. Ogihara, Y. Makita, A. Hata, M. Yamada, N. Takahashi, N. Hirawa, S. Umemura, T. Miki, M. Soma, Mutation of the folliclestimulating hormone receptor gene 5'-untranslated region associated with female hypertension. Hypertension 48, 512-518 (2006)

4. A. Ferlin, C. Vinanzi, R. Selice, A. Garolla, A.C. Frigo, C. Foresta, Toward a pharmacogenetic approach to male infertility: polymorphism of follicle-stimulating hormone $b$-subunit promoter. Fertil. Steril. 96, 1344-1349 (2011)

5. F. Tüttelmann, M. Laan, M. Grigorova, M. Punab, S. Sõber, J. Gromoll, Combined effects of the variants FSHB $-211 \mathrm{G}>\mathrm{T}$ and FSHR 2039A $>$ G on male reproductive parameters. J. Clin. Endocrinol. Metab. 97(10), 3639-3647 (2012) 
6. M. Simoni, L. Casarini, Mechanisms in endocrinology: Genetics of FSH action: a 2014-and-beyond view. Eur. J. Endocrinol. 170 (3), 91-107 (2014)

7. L. Sun, Y. Peng, A.C. Sharrow, J. Iqbal, Z. Zhang, D.J. Papachristou, S. Zaidi, L.L. Zhu, B.B. Yaroslavskiy, H. Zhou, A. Zallone, M.R. Sairam, T.R. Kumar, W. Bo, J. Braun, L. CardosoLanda, M.B. Schaffler, B.S. Moonga, H.C. Blair, M. Zaidi, FSH directly regulates bone mass. Cell 125, 247-260 (2006)

8. L. Juel Mortensen, M. Lorenzen, N. Jørgensen, A.M. Andersson, J.E. Nielsen, L.I. Petersen, B. Lanske, A. Juul, J.B. Hansen, M. Blomberg Jensen, Possible link between FSH and RANKL release from adipocytes in men with impaired gonadal function including Klinefelter syndrome. Bone 123, 103-114 (2019)

9. D. Rendina, F. Gianfrancesco, G. De Filippo, D. Merlotti, T. Esposito, A. Mingione, R. Nuti, P. Strazzullo, G. Mossetti, L. Gennari, FSHR gene polymorphisms influence bone mineral density and bone turnover in postmenopausal women. Eur. J. Endocrinol. 163, 165-172 (2010)

10. V. Barresi, S.S. Signorelli, N. Musso, M. Anzaldi, V. Fiore, M. Alberghina, D.F. Condorelli, ICAM-1 and SRD5A1 gene polymorphisms in symptomatic peripheral artery disease. Vasc. Med. 19(3), 175-181 (2014)

11. S. Sudo, M. Kudo, S. Wada, O. Sato, A.J. Hsueh, S. Fujimoto, Genetic and functional analyses of polymorphisms in the human FSH receptor gene. Mol. Hum. Reprod. 8(10), 893-899 (2002)

12. J. Gromoll, M. Simoni, Genetic complexity of FSH receptor function. Trends Endocrinol. Metab. 16(8), 368-373 (2005)

13. L. Tamburino, S. La Vignera, V. Tomaselli, R.A. Condorelli, R. Cannarella, L.M. Mongiò̀, A.E. Calogero, The -29G/A FSH receptor gene polymorphism is associated with higher FSH and LH levels in normozoospermic men. J. Assist. Reprod. Genet. 34 (10), 1289-1294 (2017)

14. P. Liu, Y. Ji, T. Yuen, E. Rendina-Ruedy, V.E. De Mambro, S. Dhawan, W. Abu-Amer, S. Izadmehr, B. Zhou, A.C. Shin, R. Latif, P. Thangeswaran, A. Gupta, J. Li, V. Shnayder, S.T. Robinson, Y.E. Yu, X. Zhang, F. Yang, P. Lu, Y. Zhou, L.L. Zhu, D.J. Oberlin, T.F. Davies, M.R. Reagan, A. Brown, T.R. Kumar, S. Epstein, J. Iqbal, N.G. Avadhani, M.I. New, H. Molina, J.B. van Klinken, E.X. Guo, C. Buettner, S. Haider, Z. Bian, L. Sun, C. J. Rosen, M. Zaidi, Blocking FSH induces thermogenic adipose tissue and reduces body fat. Nature. 546(7656), 107-112 (2017)

15. R. Zhang, S. Zhang, X. Zhu, Y. Zhou, X. Wu, Follicle-stimulating hormone receptor (FSHR) in Chinese alligator, Alligator sinensis: molecular characterization, tissue distribution and mRNA expression changes during the female reproductive cycle. Anim. Reprod. Sci. 156, 40-50 (2015)

16. N.G. Kondegowda, R. Fenutria, I.R. Pollack, M. Orthofer, A. Garcia-Ocaña, J.M. Penninger, R.C. Vasavada, Osteoprotegerin and denosumab stimulate human beta cell proliferation through inhibition of the receptor activator of NF- $\mathrm{KB}$ ligand pathway. Cell. Metab. 22(1), 77-85 (2015)

17. E. Passeri, S. Benedini, E. Costa, S. Corbetta, A. Single, $60 \mathrm{mg}$ dose of denosumab might improve hepatic insulin sensitivity in postmenopausal nondiabetic severe osteoporotic women. Int. J. Endocrinol. 2015, 352858 (2015)

18. A. Lasco, N. Morabito, G. Basile, M. Atteritano, A. Gaudio, G.M. Giorgianni, E. Morini, B. Faraci, F. Bellone, A. Catalano,
Denosumab inhibition of RANKL and insulin resistance in postmenopausal women with osteoporosis. Calcif. Tissue Int. 98(2), 123-128 (2016)

19. R. Cannarella, T. Mattina, R.A. Condorelli, L.M. Mongiò̀, G. Pandini, S. La Vignera, A.E. Calogero, Chromosome 15 structural abnormalities: effect on the IGF1R gene expression and function. Endocr. Connect. 6(7), 528-539 (2017)

20. R. Cannarella, F. Mancuso, R.A. Condorelli, I. Arato, L.M. Mongioì, F. Giacone, C. Lilli, C. Bellucci, S. La Vignera, R. Calafiore, G. Luca, A.E. Calogero, Effects of GH and IGF1 on basal and FSH-modulated porcine sertoli cells in vitro. J. Clin. Med. 8(6), E811 (2019). https://doi.org/10.3390/jcm8060811

21. R. Cannarella, I. Arato, R.A. Condorelli, G. Luca, F. Barbagallo, A. Alamo, C. Bellucci, C. Lilli, S. La Vignera, R. Calafiore, F. Mancuso, A.E. Calogero, The IGF1 receptor is involved in folliclestimulating hormone signaling in porcine neonatal sertoli cells. J. Clin. Med. 8(5), E577 (2019). https://doi.org/10.3390/jcm8050577

22. M. Honma, S. Sawada, Y. Ueno, K. Murakami, T. Yamada, J. Gao, S. Kodama, T. Izumi, K. Takahashi, S. Tsukita, K. Uno, J. Imai, E. Kakazu, Y. Kondo, K. Mizuno, N. Kawagishi, T. Shimosegawa, H. Katagiri, Selective insulin resistance with differential expressions of IRS- 1 and IRS-2 in human NAFLD livers. Int. J. Obes. 42(9), 1544-1555 (2018)

23. H. Shakeri, A. Khoshi, M. Kaffash Bajestani, A. Farahi, M.S. Javadzadeh, Z. Hosseini, R. Mohammadi, Association of IRS1 GLY971ARG gene polymorphism with insulin resistance in Iranian newly diagnosed diabetic adults. Acta Endocrinol. 15(3), 317-322 (2019)

24. N.C. Law, M.E. Hunzicker-Dunn, Insulin receptor substrate 1, the hub linking follicle-stimulating hormone to phosphatidylinositol 3-kinase activation. J. Biol. Chem. 291, 4547-4560 (2016)

25. R. Cannarella, R.A. Condorelli, S. La Vignera, A.E. Calogero, Effects of the insulin-like growth factor system on testicular differentiation and function: a review of the literature. Andrology 6 (1), 3-9 (2018)

26. R. Cannarella, I. Arato, R.A. Condorelli, L.M. Mongioì, C. Lilli, C. Bellucci, S. La Vignera, G. Luca, F. Mancuso, A. E. Calogero, Effects of insulin on porcine neonatal sertoli cell responsiveness to FSH in vitro. J. Clin. Med. 8(6), 809 (2019). https://doi.org/10. 3390/jcm8060809

27. P. Xu, B.Y. Huang, J.H. Zhan, M.T. Liu, Y. Fu, Y.Q. Su, Q.Y. Sun, W.H. Wang, D.J. Chen, J.Q. Liu, Insulin reduces reaction of follicular granulosa cells to FSH stimulation in women with obesity-related infertility during IVF. J. Clin. Endocrinol. Metab. 104(7), 2547-2560 (2019)

28. R. Cannarella, S. La Vignera, R.A. Condorelli, L.M. Mongioì, A. E. Calogero, FSH dosage effect on conventional sperm parameters: a meta-analysis of randomized controlled studies. Asian J. Androl. (2019). https://doi.org/10.4103/aja.aja_42_19

29. S. La Vignera, R.A. Condorelli, Y. Duca, R. Cannarella, F. Giacone, A.E. Calogero, FSH treatment for normogonadotropic male infertility: a synergistic role for metformin? Eur. Rev. Med. Pharmacol. Sci. 23(13), 5994-5998 (2019)

30. V. Pergialiotis, A. Prodromidou, M. Frountzas, L.M. Korou, G.D. Vlachos, D. Perrea, Diabetes mellitus and functional sperm characteristics: a meta-analysis of observational studies. J. Diabetes Complicat. 30(6), 1167-1176 (2016) 